\title{
Near-infrared Fourier transform room-temperature photoluminescence of erbium complexes
}

\author{
J. Barbillat ${ }^{\mathrm{a})}$ \\ LASIR CNRS, USTL Bât C5, 59655 Villeneuve d'Ascq, France \\ P. Le Barny, L. Divay, E. Lallier, and A. Grisard \\ Thales Research and Technology-France, Domaine de Corbeville, 91404 Orsay, France \\ R. Van Deun and P. Fias \\ K. U. Leuven, Department of Chemistry, Coordination Chemistry Division, Celestijnenlaan 200F, \\ 3001 Leuven (Heverlee), Belgium
}

(Received 5 March 2003; accepted 29 July 2003)

\begin{abstract}
A modified Fourier transform (FT) Raman bench spectrometer designed for the detection of weak light emission in the $800-1700 \mathrm{~nm}$ wavelength region has been used to demonstrate the advantages of FT spectroscopy for measuring near-infrared photoluminescence spectra of lanthanide complexes with a good resolution and very good sensitivity. This apparatus has been tested with an ultraviolet laser source $(325 \mathrm{~nm})$ on three standard erbium complexes. The ${ }^{4} I_{13 / 2} \rightarrow{ }^{4} I_{15 / 2}$ emission of tris-(acetylacetonato) (1,10 phenanthroline) erbium $\left[\operatorname{Er}(\mathrm{acac})_{3}(\mathrm{phen})\right]$, tris- $(4,4,4,-$ trifluoro-1-(2 thenoyl)-1,3-butenedione $) \quad(1,10$ phenanthroline $)$ erbium $\left[\operatorname{Er}(T T F A)_{3}(\right.$ phen $\left.)\right]$ and tris(8-hydroxyquinolinato) erbium $\left[\mathrm{Erq}_{3}\right]$ has thus been recorded in solution and in the solid state and compared with literature. (C) 2003 American Institute of Physics. [DOI: 10.1063/1.1614873]
\end{abstract}

Amplification of telecommunication signals required for a variety of loss compensated components is currently achieved using well-established planar integrated optics technology based on erbium doped glass or silica-on silicon. ${ }^{1,2}$ This is because the $\mathrm{Er}^{3+}$ ion shows a sharp luminescence in the third telecommunication window (centered at $1540 \mathrm{~nm}$ ) due to an intra- $4 f$ shell transition from its first excited state $\left({ }^{4} I_{13 / 2}\right)$ to the ground state $\left({ }^{4} I_{15 / 2}\right)$. However, the full width at half maximum (FWHM) of any Er-doped inorganic material is relatively small. As an example, Erimplanted silica has a typical FWHM value of $11 \mathrm{~nm}$ for the most intense peak. The strong demand for increased bandwidth has generated a lot of interest for Er organic compounds $^{3}$ (Er complexes) since they exhibit broad luminescence spectra with a FWHM of $70 \mathrm{~nm}^{4}$ Furthermore, Er complexes, which are in principle compatible with polymers, allow to take advantage of the plastic optical fibers technology, and hence, open the possibility to achieve low cost all plastic components for telecommunications. One of the stumbling blocks is to avoid luminescence quenching which is due to coupling to vibrational states of the $\mathrm{C}-\mathrm{H}$ and $\mathrm{O}-\mathrm{H}$ bonds in the complexes. The approach which is currently followed to get rid of the quenching issue is to use perfluorinated and/or perdeuterated ligands.

One of the first characterizations of such Er complexes is the recording of their near-infrared (NIR) fluorescence spectra. As no NIR fluorimeters are commercially available, homemade experimental apparatus are currently used. As the signal to be detected is still weak, the sensitivity of the apparatus has to be very high. We have used the advantages of

\footnotetext{
a) Author to whom correspondence should be addressed; electronic mail: jacques.barbillat@univ-lille1.fr
}

Fourier transform (FT) spectroscopy to record the NIR photoemission from erbium complexes. In this spectral region, the interferometer outperforms the grating spectrometer giving a better signal-to-noise ratio (SNR) for the same total acquisition time. Moreover, high spectral resolution is much easier to attain using an interferometer-based instrument. The interferometer offers major improvements in light throughput and data acquisition. With an interferometer, the optical throughput is increased and an improvement in the sensitivity is obtained as circular apertures replace the slits (Jacquinot's advantage).

Second, as the detector simultaneously collects the entire spectral region, the noise is distributed throughout the transformed spectrum resulting in an increase in the SNR, if the noise is random and independent of the signal intensity as it is the case in the NIR region (Fellgett's advantage). Other advantages are the very high precision of the wavelength axis (Connes's advantage) which results from the use of an internal $\mathrm{He}-\mathrm{Ne}$ reference laser allowing wavelength calibration, rapid signal acquisition times, and improved spectral resolution.

Three standard Er complexes have been chosen to validate this approach (Fig. 1): tris-(acetylacetonato) (1,10 phenanthroline) erbium $\left[\operatorname{Er}(\mathrm{acac})_{3}(\mathrm{phen})\right]$, tris- $(4,4,4,-$ trifluoro-1-(2 thenoyl)-1,3-butenedione (1,10 phenanthroline) erbium $\left[\operatorname{Er}(\mathrm{TTFA})_{3}\right.$ (phen) $]$, and tris(8-hydroxyquinolinato) erbium $\left[\mathrm{Erq}_{3}\right]$.

$\left[\operatorname{Er}(\mathrm{acac})_{3}(\mathrm{phen})\right]$ and $\left[\operatorname{Er}(\mathrm{TTFA})_{3}(\mathrm{phen})\right]$ were prepared by refluxing erbium (III) chloride with stoichiometric amounts of convenient ligands in ethanol. [ $\left.\operatorname{Er}(\mathrm{acac})_{3}(\mathrm{phen})\right]$ preparation required a further treatment with a diluted solution of sodium hydroxide. $\left[\mathrm{Erq}_{3}\right]$ was obtained by adding $1 \%$ 8-hydroxyquinoline in alcohol to erbium(III) nitrate in an 


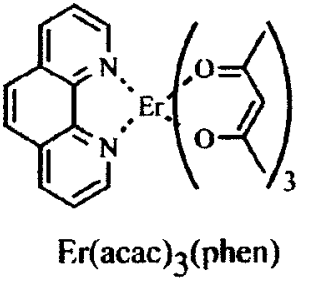

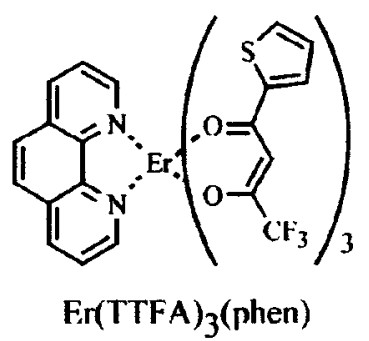

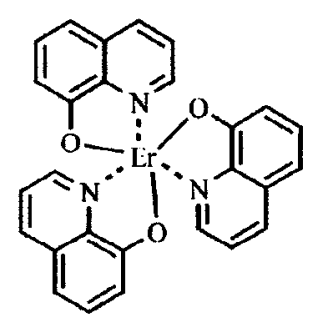

$\mathrm{Erq}_{3}$

FIG. 1. Chemical structure of the erbium complexes under study.

aqueous solution heated at $60^{\circ} \mathrm{C}$. By adding a few drops of ammonia the complex precipitated. The three complexes were purified by crystallization in ethanol.

All measurements were performed with a highly sensitive Bruker FRA 106 FT Raman module (adapted to an IFS 88 FTIR bench spectrometer) initially designed for the detection, in the $800-1700 \mathrm{~nm}$ wavelength region, of weak scattered radiation upon NIR laser excitation (Fig. 2). The Raman module has been modified for accepting external ultraviolet (UV) laser source $(325 \mathrm{~nm})$ instead of the internal Nd:yttrium-aluminum-garnet (YAG) NIR laser source at $1064 \mathrm{~nm}$ in order to measure laser induced NIR luminescence of erbium complexes instead of scattered radiation. ${ }^{5}$

This instrument is fitted with an ultrasensitive Germanium detection system (model EO-817L: North Coast Scientific Co. Santa Rosa, CA).

The detector comprises a Ge pin photodiode element (5 $\mathrm{mm}$ in diameter), an integral preamplifier and a liquid nitrogen dewar. Ultrapure Ge pin diode detection system offers unparalleled responsivity and noise equivalent power (NEP) performance for the spectral range $800-1700 \mathrm{~nm}$ since this

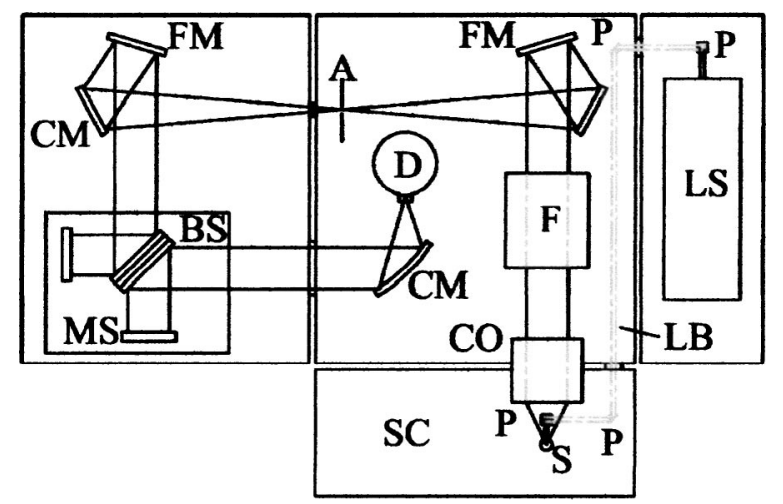

FIG. 2. FT Raman bench spectrometer before modification. SC: sample chamber, $S$ : sample, $P$ : prism, LS: laser source, LB: laser beam, CO: collection optics, $F$ : filter, FM: flat mirror, CM: concave mirror, $D$ : detector, $A$ : aperture, BS: beam splitter, MS: mirror scanner.

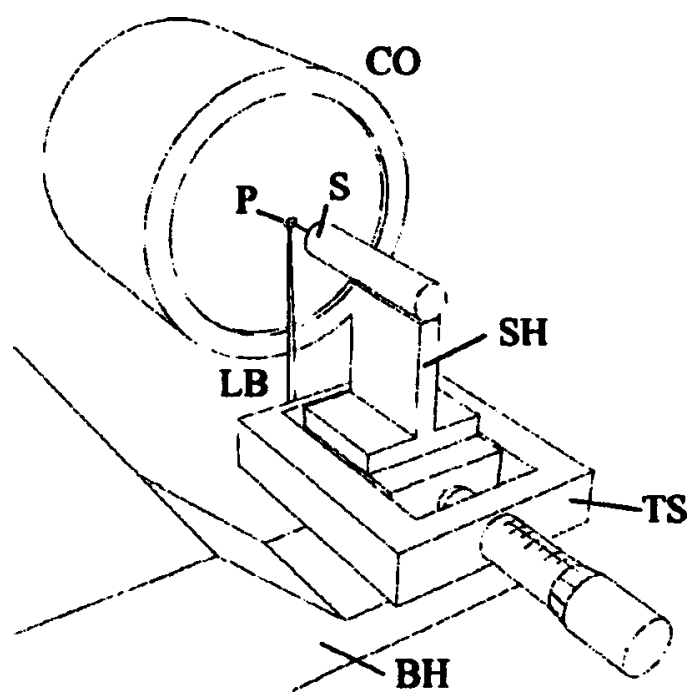

FIG. 3. FT Raman sample chamber. SH: sample holder, BH: base holder, TS: translation stage.

device exhibits negligible diffusion, injection, and surface leakage currents. Its noise is primarily due to the statistical generation of photocarriers by background radiation in the $1500 \mathrm{~nm}$ region. Precise temperature adjustment and control of principal noise sources in the preamplifier electronics are key elements for minimizing noise contribution of the preamplifier stage. The result is an overall NEP of $10^{-15} \mathrm{~W} \mathrm{~Hz}^{-1 / 2}$.

The laser source used for the excitation of the erbium photoluminescence is a continuous wave $(\mathrm{He}-\mathrm{Cd})$ laser emitting about $35 \mathrm{~mW}$ at $325 \mathrm{~nm}$ (Kimmon, IK $3351 \mathrm{R}-\mathrm{G}$ ). Before entering the sample compartment, the laser emission was filtered by means of a short-wave pass colored filter transmitting more than $66 \%$ of the laser beam at $325 \mathrm{~nm}$ and eliminating most NIR radiations from the laser discharge tube around $1500 \mathrm{~nm}$. Since, the Nd-YAG laser source is included in a special compartment of the spectrometer and the optical path (underneath the spectrometer) for the NIR laser beam involves several glass prisms before entering the sample chamber (Figs. 2 and 3), we were obliged to mount the UV laser outside the spectrometer and to modify the sample illumination scheme.

We have recorded luminescence spectra of both erbium complexes in DMF solutions and erbium powders samples. All spectra were obtained at room temperature using the high numerical aperture collection optics of the FT-Raman module. Erbium solutions were placed in a quartz cuvette and the photoluminescence signal was collected at right angle from the incident laser beam (Fig. 4). For this configuration the regular sample holder has been replaced by a base plate supporting the quartz cuvette, a steering mirror, and a quartz focusing lens. The lens is mounted on a translation stage in order to adjust the focusing of the laser beam exactly at the center of the cuvette. The base plate is also mounted on another translation stage for adjusting the illuminated region of the sample at the focal plane of the collection optics. Solid samples were studied as small pellets just pressed on a metal substrate mounted on the standard sample holder and the luminescence signal was collected at $180^{\circ}$ from the incident 


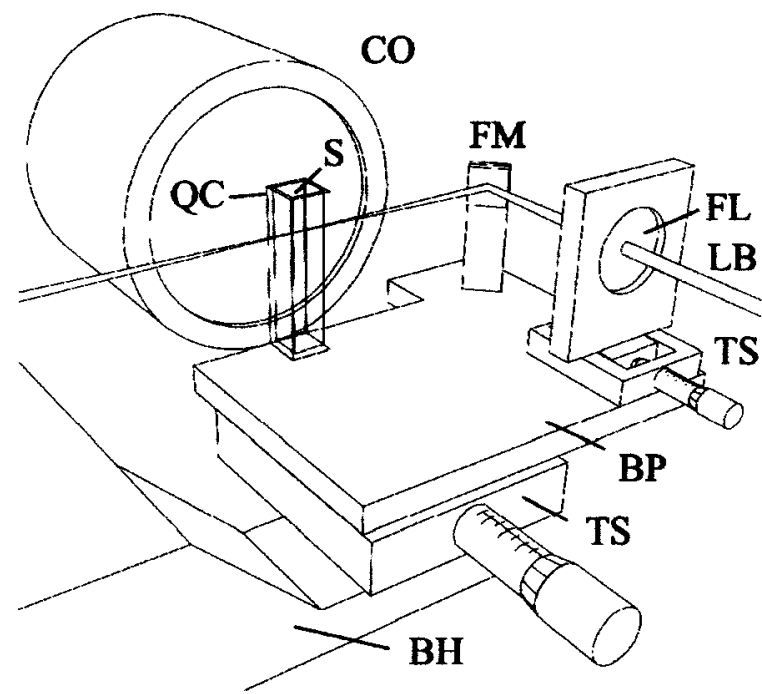

FIG. 4. Apparatus for the study of Er complexes in solution with an external UV laser. QC: quartz cuvette: FL: focusing lens, BP: base plate.

laser beam in the so-called backscattered configuration (Fig. 5). In this configuration the UV laser beam is focused onto the sample using a quartz focusing lens, a steering mirror, and the small prism located in front of the collection optics. This prism must be turn $90^{\circ}$ with respect to its regular position for NIR Raman measurements where the laser beam goes upwards from underneath the spectrometer (Fig. 3). Here again the focusing lens and the sample holder can be moved independently for optimizing the focalization of the laser beam onto the sample and the collection of the luminescence radiation.

Luminescence signal around $1500 \mathrm{~nm}$ passes unaffected through the notch filter $(F)$ used for rejecting the $1064 \mathrm{~nm}$ Nd-YAG laser emission in standard FT-Raman measurements (this filter exhibits a cutoff region of about $23 \mathrm{~nm}$ centered at $1064 \mathrm{~nm}$ ). Moreover, a long-wave pass silicon filter in front of the Ge detector eliminates all visible wavelengths below $1050 \mathrm{~nm}$ that could disturb the detection of the luminescence emission.

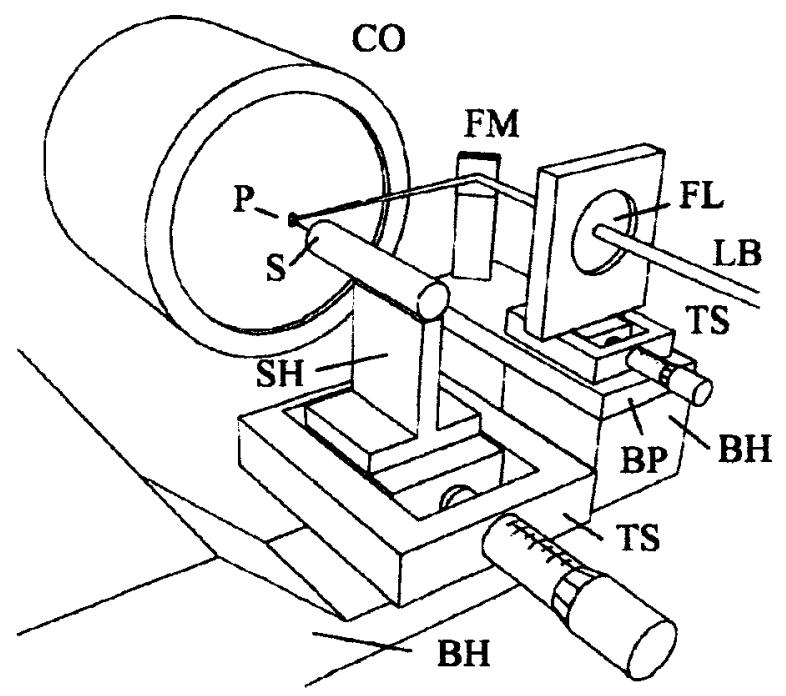

FIG. 5. Apparatus for the study of Er complexes in the solid state with an external UV laser.

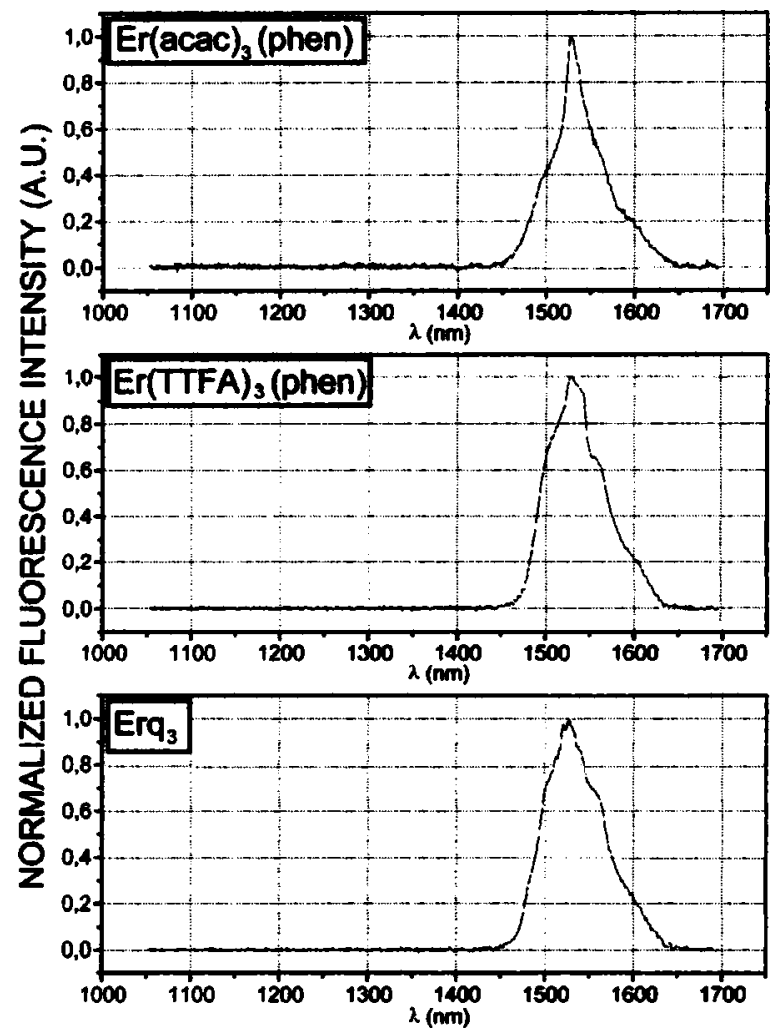

FIG. 6. NIR photoluminescence spectra of Er complexes in DMF solution. $\lambda_{\mathrm{exc}}=325 \mathrm{~nm}$.

Each experiment required to acquire the data approximately 4 min (200 scans at $4 \mathrm{~cm}^{-1}$ resolution) for Er solutions and $1 \mathrm{~min}\left(50\right.$ scans at $4 \mathrm{~cm}^{-1}$ resolution) for samples in the solid state. The spectral resolution of $4 \mathrm{~cm}^{-1}$ (about 1 $\mathrm{nm}$ at $1500 \mathrm{~nm}$ ) is sufficient to observe the characteristic broad spectral features of the samples in the region 1450$1650 \mathrm{~nm}$ with a fairly good SNR. Figures 6 and 7 show the photoluminescence spectra of Er complexes in solution and in the solid state, respectively. In each case the ligand absorption is followed by an energy transfer to $\mathrm{Er}^{3+}$ which emits finally in the NIR via the ${ }^{4} I_{13 / 2} \rightarrow{ }^{4} I_{15 / 2}$ transition. The SNR of these spectra ranges from 182 to 347 for the liquid samples and from 117 to 148 for the samples in the solid state.

The difference between the solution and the solid state measurements could be explained by a different chemical environment in solid state and in solution of the complexes. Dilute solution experiments give rise to the isolated molecule spectrum whereas solid state experiments lead to information concerning the collective behavior of the molecules. Moreover the solution measurements have been done in a coordinating solvent (DMF). This solvent can coordinate with the Er ion, and hence, influence the fine structure of the luminescence signal. It would be very difficult, however, to explain the shape and the fine structure of the signal in great detail. In our case, bulk material spectral features were interesting for device applications where high concentration is required, while solution measurements were necessary to compare with literature data.

The obtained NIR photoluminescence spectra of 


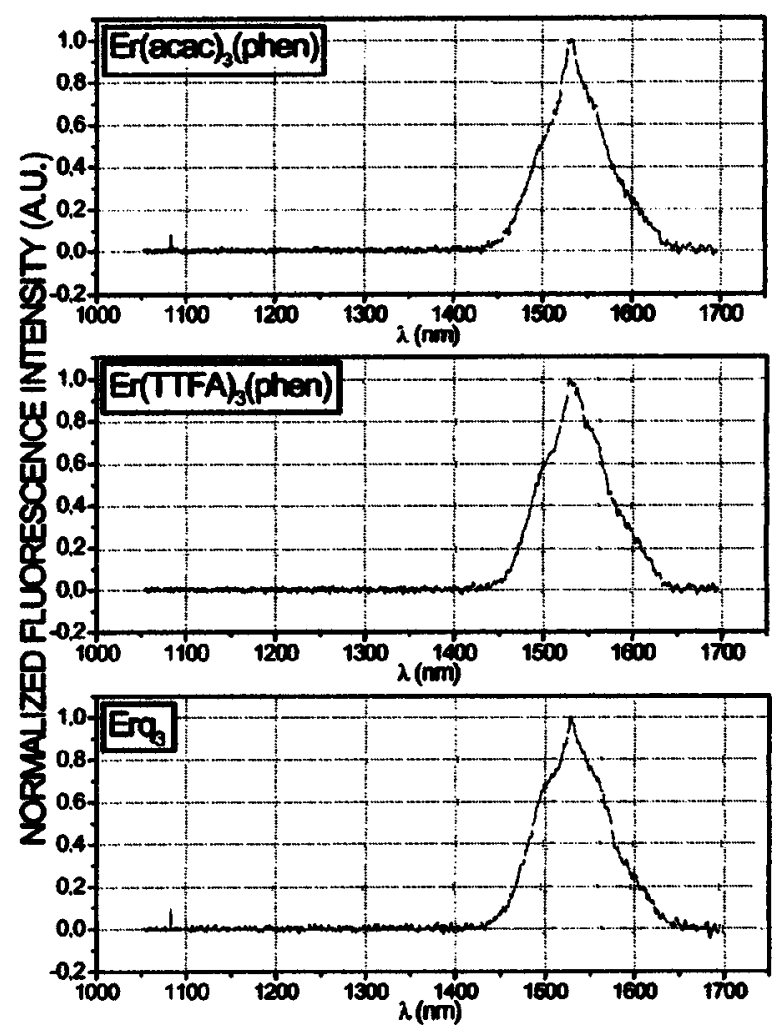

FIG. 7. NIR photoluminescence spectra of Er complexes in the solid state. $\lambda_{\text {exc }}=325 \mathrm{~nm}$.

$\left[\operatorname{Er}(\mathrm{acac})_{3}(\right.$ phen $\left.)\right]$ and $\left[\mathrm{Erq}_{3}\right]$ are consistent with those already published. ${ }^{6,7}$ In particular, the slight differences previously observed ${ }^{6,7}$ between the shapes of the $\left[\operatorname{Er}(\mathrm{acac})_{3}(\mathrm{phen})\right]$ and $\left[\mathrm{Erq}_{3}\right]$ photoluminescence spectra have been well recognized. The influence of the ligands on the feature of the photoluminescence spectra is clearly reduced in the solid state. However, since the experimental apparatus described in this paper cannot yet allow the fluorescence quantum yield measurement, we cannot directly compare our results with those of literature. Nevertheless, future improvements of the apparatus including an integrating sphere will allow us to estimate quantum yields in a very reliable way.

In conclusion, we have demonstrated that a modified FT Raman spectrometer allows to record NIR photoluminescence of lanthanide complexes with a good resolution. This provides an efficient tool for characterizing NIR emitting molecules even with a very low quantum yield fluorescence efficiency.

The authors would like to thank Laetitia Pierron for her help in preparing and purifying some Er complexes, Professor J. Delaire for helpful discussion and Jobin\&Yvon (Horiba group) for the loan of the $\mathrm{He}-\mathrm{Cd}$ laser source. This work was supported by the European community under Contract GROWTH No: GRD2-2000-30346 OPAMD.

\footnotetext{
${ }^{1}$ A. Polman, Mater. Res. Soc. Symp. Proc. 597, 3 (2000).

${ }^{2}$ M. N. Zervas and G. van den Hoven, Photonics 4, 151 (2001).

${ }^{3}$ K. Kuriki, Y. Koike, and Y. Okamoto, Chem. Rev. (Washington, D.C.) 102, 2347 (2002).

${ }^{4}$ L. H. Slooff, A. Polman, M. P. Oude Wolbers, F. C. J. M. van Veggel, D. Reinhoudt, and J. W. Hofstraat, J. Appl. Phys. 83, 497 (1998).

${ }^{5}$ J. M. Wessels, P. Charlesworth, and M. A. Rodgers, Photochem. Photobiol. 61, 350 (1995).

${ }^{6}$ R. G. Sun, Y. Z. Wang, Q. B. Zheng, H. J. Zhang, and A. J. Epstein, J. Appl. Phys. 87, 7589 (2000).

${ }^{7}$ W. P. Gillin and R. J. Curry, Appl. Phys. Lett. 75, 1380 (1999).
} 